\title{
Postpartum Opioid Use Following Vaginal Deliveries with No or Minor Obstetric Perineal Lacerations
}

\author{
Samantha Richardson, MD' ${ }^{1}$,Todd W. Gress, MD'1, \\ Amanda Pauley, MD' ${ }^{1}$, David C. Jude, MD ${ }^{1}$
}

ABSTRACI

Author affiliations are

listed at the end of this

BACKGROUND: Recommendations for management of pain following vaginal delivery are not specific and do not differentiate patients based on the degree of perineal trauma. Our objective is to describe patterns of postpartum opioid use in women with no or minor perineal trauma.

METHODS: This is a retrospective cohort study describing the characteristics of opioid analgesia usage among women with no or minor perineal lacerations at the time of vaginal delivery in a university affiliated regional hospital.

REsULTS: For the 6-month study period, 433 patients were eligible for inclusion. Of these, $423(97.69 \%)$ were ordered as needed narcotics during their post-partum hospitalization. Of women with an as needed narcotic prescribed, $285(65.82 \%)$ used at least one dose of narcotics while hospitalized. Significant patient characteristics of women using opioids during their inpatient post-partum course included those who used epidural analgesia during labor $(p=0.009)$ and primiparous patients $(p=0.05)$. Thirty-five of the women included in the study received a prescription for opioid analgesics at the time of discharge (8.08\%). Significant findings among these women include increasing maternal age $(p=0.007)$. Non-academic physicians with resident coverage were 3.1 times more likely to prescribe opioids at discharge compared to academic physicians with residents and non-academic physicians without resident coverage.

ConcLusions: Focusing specifically on women with no or minor perineal lacerations at the time of delivery, our findings indicate that if given the option of opioids analgesia during their hospitalization, many women will request at least one dose of opioid analgesia, but rarely require opioids after discharge.

\section{KEYWORDS}

Postpartum analgesia, Obstetric laceration, Opioid analgesia, Vaginal delivery

\section{INTRODUCTION}

The opioid epidemic has grown substantially in the United States over the past twenty years. The 2015 National Survey on Drug Use and Health estimated that 91.8 million (37.8\%) of U.S. civilian, noninstitutionalized adults used prescription opioids; 11.5 million (4.7\%) misused them; 1.9 million $(0.8 \%)$ had a substance use disorder. ${ }^{1}$ Abuse of opioid pain relievers is a particular problem in reproductiveage women. In the United States, overdose deaths increased more than fivefold among women between 1999 and $2010 .^{2}$ In 2016, there were eighteen maternal deaths in West Virginia, of which 44\% either had a documented substance use disorder or died from an overdose. ${ }^{3}$ Although the number of opioids prescribed in the United States has decreased since 2010 , there are currently roughly three times more prescriptions for opioids than in 1999.4 The rate of opiate prescribing for West Virginia ranks as the thirdhighest in the nation. ${ }^{5}$ Prabhu et al discovered that approximately 850,000 women are prescribed opiates following vaginal deliveries each year and suggest that opiates are overprescribed in this patient population. ${ }^{6}$ While opiate use in patients after vaginal delivery may sometimes be necessary, opiate use 
in this population is also complicated by the central nervous system depression observed in neonates breastfed by mothers receiving opioid analgesia. ${ }^{7}$

The Joint Commission requires accredited hospitals to establish policies and procedures that address comprehensive clinical assessment of pain, as well as to minimize risks associated with opiate use. ${ }^{8}$ Obstetric care providers simultaneously are asked to keep their patients comfortable without overprescribing opioid analgesics. However, evidence-based guidance is limited. There is little information related to opioid analgesia recommendations in this patient population, especially for women with no or minor perineal trauma. The American College of Obstetricians and Gynecologists (ACOG) recommends a multimodal pain control approach with neuraxial analgesia, nonsteroidal anti-inflammatory drugs (NSAIDs), and acetaminophen. There is no recommendation for opiate use after vaginal delivery. ${ }^{9}$ Likewise, the American Pain Society, the American Society of Regional Anesthesia and Pain Medicine, as well as the American Society of Anesthesiologists, recommend general postoperative pain guidelines including multimodal analgesia, adjustment of pain management based on the adequacy of pain relief, and patient education regarding pain relief expectations post-procedure. ${ }^{10}$ Acetaminophen and non-steroidal anti-inflammatory drugs are both equally effective at decreasing the need for opiate analgesia. ${ }^{11}$

Since there is little guidance to clinicians regarding how to manage post-delivery pain for women with no or minor perineal lacerations following vaginal delivery, we sought to investigate the patterns of inpatient prescribing, inpatient use, and discharge prescribing practices of opioids in this patient population. The large number of women delivering annually with little or no perineal trauma makes studying approaches to postpartum analgesia crucial.

\section{METHODS}

This is a retrospective descriptive study focusing on inpatient opioid use and outpatient narcotic prescribing practices in women with no or minor perineal lacerations following vaginal delivery at
Cabell Huntington Hospital $(\mathrm{CHH})$ in Huntington, WV. No obstetric laceration is self-descriptive to include those women who delivered with both an intact perineum and no vaginal mucosal lacerations. For the purposes of this study, we defined minor perineal lacerations to include firstdegree laceration, periurethral or labial tears. $\mathrm{CHH}$ is a university-affiliated regional hospital and, for the study year, was the second busiest delivering hospital in the state of West Virginia performing 2,561 obstetrical deliveries during the calendar year 2017. At the study hospital, there are deliveries by midwives, family practice physicians, private practice obstetricians without resident coverage, private practice obstetricians with resident coverage, and academic obstetricians with residents.

We reviewed all vaginal deliveries from July 2017 through December 2017. We included all deliveries resulting in no or minor perineal lacerations as described above. Breech presentation, multiple gestations, known current opiate abuse, opioid dependence with current enrollment in a medication-assisted treatment (MAT) program, intrauterine fetal demise, and women under age 18 were excluded from the study. Patients receiving local and pudendal nerve blocks were also excluded.

In our medical review, patient information gathered included the following: maternal age; maternal race; gestational age; gravidity; parity; operative vs. spontaneous delivery; degree of perineal trauma; intrapartum analgesia; provider status; inpatient opiate prescribing; inpatient opiate use; and discharge opiate prescriptions including the number of tablets. We evaluated the study group based on the degree of perineal trauma as follows: intact perineum with no mucosal lacerations, periurethral or labial lacerations, or first-degree laceration. Intrapartum analgesia was evaluated based on the use of epidural analgesia. Parity was separated into nulliparous and multiparous women. Provider status was assessed based on the following: family practice physician, private practice physician without residents, certified nurse-midwife, private practice physician with residents, or academic physician with residents.

We performed bivariable analysis using the Student's 


\begin{tabular}{|l|l|l|l|}
\hline Characteristics & $\begin{array}{l}\text { Inpatient Opioid } \\
\text { Use } \\
(\mathrm{N}=285)^{*}\end{array}$ & $\begin{array}{l}\text { No Inpatient Opioid } \\
\text { Use } \\
\text { (N=138) }\end{array}$ & $\begin{array}{l}\text { P- } \\
\text { Value }\end{array}$ \\
\hline Maternal age, mean (sd) & $25.9(0.32)$ & $26.8(0.44)$ & 0.10 \\
\hline Caucasian Race, \% & $92.3(263)$ & $89.9(124)$ & 0.10 \\
\hline Gravity, mean (sd) & $2.8(0.10)$ & $2.7(0.12)$ & 0.52 \\
\hline Parity, mean (sd) & $1.2(0.07)$ & $1.4(0.09)$ & 0.32 \\
\hline Nulliparous, \% & $26.0(74)$ & $17.4(24)$ & 0.05 \\
\hline Spontaneous Delivery, \% & $97.9(279)$ & $98.6(136)$ & 0.64 \\
\hline Epidural, \% & $93.3(266)$ & $85.5(118)$ & 0.009 \\
\hline Provider Status, \% & & & \\
Private Physician with Residents & $57.9(165)$ & $47.1(65)$ & 0.29 \\
Academic Physician with Residents & $26.7(76)$ & $34.1(47)$ & \\
Private Physician without Residents & $10.9(31)$ & $13.8(19)$ & \\
Certified Nurse Midwife & $3.5(10)$ & $4.4(6)$ & \\
Academic Family Physician & $1.0(3)$ & $0.72(1)$ & \\
\hline Minor perineal Trauma, \% & & $24.6(34)$ & \\
First Degree Laceration & $28.4(81)$ & $13.8(19)$ & \\
Periurethral Laceration & $11.2(32)$ & $61.6(85)$ & 0.005 \\
\hline Intact Perineum & $60.4(172)$ & $2.9(4)$ & \\
\hline Opioid Prescription on Discharge, \% & $10.9(31)$ & .900 & \\
\hline
\end{tabular}

*Numbers in parentheses represent the total $\mathrm{N}$ unless otherwise indicated

TABLE 1. Characteristics of 423 Patients undergoing Vaginal Delivery with Minor Perineal Trauma by Inpatient Opioid Use

T-test for continuous variables and Chi-square or Fisher's exact when appropriate for categorical variables. We examined the association of patients receiving an opioid prescription at discharge by multiple logistic regression. All analyses were performed using Stata 15.0 (College Station, TX). The Marshall University Institutional Review Board approved the study. This research did not receive any specific grant from funding agencies in the public, commercial, or not-for-profit sectors.

\section{RESULTS}

Between July 2017 through December 2017, 484 vaginal deliveries met our inclusion criteria. Thirty patients were excluded due to illicit opiate use or enrollment in a MAT program, twelve were excluded due to maternal age, one delivery was excluded due to multiple gestations, three were excluded due to local anesthesia use at the time of delivery, four were excluded due to pudendal block anesthesia, and one was excluded due to intrauterine fetal demise, leaving a sample size of 433 patients. There were no breech vaginal deliveries during the time oral opioid analgesic in the hospital. Parity was the only significant difference in women using opiates in the hospital, as $26 \%$ of nulliparous women use at least one dose of opiate analgesia compared to $17.4 \%$ of multiparous patients. Of the total patient sample, there were 35 narcotic prescriptions at discharge (8.08\%), with 766 tablets and an average of 21.9 tablets per prescription.

Two hundred eighty-five patients with no or minor perineal lacerations after vaginal delivery used opiates in the hospital (Table 1). The mean maternal age was 25.9 years. The majority of patients in this sample were Caucasian (92.3\%). The mean gravidity was 2.8 , and $26.0 \%$ of these patients were primiparous. The majority (97.9\%) were spontaneous deliveries. Epidural analgesia during labor was provided to $93.3 \%$ of these patients. Obstetric provider data revealed $57.9 \%$ of subjects were delivered by private physicians with residents, $26.7 \%$ were delivered by academic physicians with residents, $10.9 \%$ were delivered by private physicians without residents, 3.5\% were delivered by certified nurse midwives, and $1.0 \%$ were delivered by academic family practice physicians. First-degree laceration was observed in $28.4 \%, 11.2 \%$ experienced periurethral or labial lacerations, and $60.4 \%$ had an intact perineum after vaginal delivery. Of the women
Marshall Journal of Medicine

Volume 6 Issue 3 


\begin{tabular}{|l|l|l|l|}
\hline Characteristics & $\begin{array}{l}\text { Opioid Prescription } \\
\text { at Discharge } \\
(\mathrm{N}=35)^{*}\end{array}$ & $\begin{array}{l}\text { No Opioid Prescription } \\
\text { at Discharge } \\
(\mathrm{N}=388)\end{array}$ & $\begin{array}{l}\text { P- } \\
\text { Value }\end{array}$ \\
\hline Maternal age, mean (sd) & $28.4(1.0)$ & $25.9(0.26)$ & 0.007 \\
\hline Caucasian Race, \% & $97.1(34)$ & $91.0(353)$ & 0.70 \\
\hline Gravity, mean (sd) & $2.7(0.21)$ & $2.8(0.08)$ & 0.75 \\
\hline Parity, mean (sd) & $1.3(0.17)$ & $1.3(0.06)$ & 0.94 \\
\hline Nulliparous, \% & $14.3(5)$ & $24.0(93)$ & 0.29 \\
\hline Spontaneous Delivery, \% & $97.1(34)$ & $98.2(381)$ & 0.50 \\
\hline Epidural, \% & $8.6(3)$ & $9.3(36)$ & 1.0 \\
\hline Provider Status, \% & & & \\
Private Physician with Residents & $77.1(27)$ & $52.3(203)$ & 0.10 \\
Academic Physician with Residents & $14.3(5)$ & $30.4(118)$ & \\
Private Physician without Residents & $8.6(3)$ & $12.1(47)$ & \\
Certified Nurse Midwife & $0(0)$ & $4.1(16)$ & $1.0(4)$ \\
Academic Family Physician & $0(0)$ & & \\
\hline Minor perineal Trauma, \% & $28.1(105)$ & 0.97 \\
First Degree Laceration & $28.6(10)$ & $12.1(47)$ & \\
Periurethral Laceration & $11.4(4)$ & $60.8(236)$ & \\
Intact Perineum & $60.0(21)$ &
\end{tabular}

*Numbers in parentheses represent the total $\mathrm{N}$ unless otherwise indicated

TABLE 2. Characteristics of 423 Patients undergoing Vaginal Delivery with Minor Perineal Trauma by Receipt of Opioid Prescription at Hospital Discharge

using opioid analgesia while hospitalized, 10.9\% received an opioid prescription at discharge.

Of the 138 patients that did not use opiates in the hospital after vaginal delivery with no or minor perineal lacerations, the mean maternal age was 26.8 years. The majority of patients in this sample were Caucasian (89.9\%). The mean gravidity was 2.7 , and $17.4 \%$ of these patients were primiparous. The majority (98.6\%) were spontaneous deliveries and $85.5 \%$ of these patients received an epidural. Obstetric provider data for women who did not use opioids demonstrated $47.1 \%$ were delivered by private physicians with residents, $34.1 \%$ were delivered by academic physicians with residents, $13.8 \%$ were delivered by private physicians without residents, $4.4 \%$ were delivered by certified nurse midwives, and $0.72 \%$ were delivered by academic family practice physicians. First-degree laceration was found in $24.6 \%, 13.8 \%$ experienced periurethral lacerations, and $61.6 \%$ had an intact perineum after vaginal delivery. There was no significant correlation between inpatient opiate use and maternal age, race, gravidity, operative vs. spontaneous delivery, provider status, or type of minor perineal trauma.
Primiparous patients were significantly more likely to use opiates as an inpatient $(P=0.05)$. Patients with epidural use were significantly more likely to use opiates as an inpatient compared to women who did not use intrapartum epidural analgesia $(\mathrm{P}=0.009)$. Patients who received narcotics as an inpatient were significantly more likely to receive an opioid prescription at discharge $(p=0.005)$.

Regarding the 35 patients receiving an opioid prescription at discharge (Table 2 ), the mean maternal age was 28.4 years. The majority of patients in this sample were Caucasian (97.1\%). The mean gravidity was 2.7 , and $14.3 \%$ of these patients were primiparous. The majority (97.1\%) were spontaneous deliveries, and $8.6 \%$ of these patients received an epidural. Provider characteristics for women receiving an opioid prescription at discharge showed that $77.1 \%$ were delivered by private physicians with residents, $14.3 \%$ were delivered by academic physicians with residents and $8.6 \%$ were delivered by private physicians without residents. There were no deliveries in this group by certified nursemidwives or academic family practice physicians. First-degree laceration was observed in $28.6 \%, 11.4 \%$ experienced periurethral or labial lacerations, and $60.0 \%$ had an intact perineum after vaginal delivery. Of the 388 patients in this sample that did not receive an opioid prescription at discharge, the mean maternal age was 25.9 years. The majority of patients in this sample were Caucasian (91.0\%). The mean gravidity was 2.8 , and $24.0 \%$ of these deliveries were primiparous. The majority (98.2\%) were spontaneous deliveries, and $9.3 \%$ of these patients received an epidural. Provider type was $52.3 \%$ delivered by private physicians with residents, $30.4 \%$ were delivered by academic physicians with residents, 
$12.1 \%$ were delivered by private physicians without residents, $4.1 \%$ were delivered by certified nurse midwives, and $1.0 \%$ were delivered by academic family practice physicians. A first-degree laceration was observed in $27.1 \%, 12.1 \%$ experienced periurethral or labial lacerations, and $60.8 \%$ had an intact perineum after vaginal delivery.

There was no significant correlation between receiving an opiate prescription at discharge and maternal race, gravidity, operative vs. spontaneous delivery, parity, use of anesthesia, or type of minor perineal trauma. Maternal age was significantly associated with receiving an opioid prescription at discharge (mean= 28.4 v. 25.9 years old) $(p=0.007)$. A small percentage of women (2.9\%) received an opioid prescription at discharge even though they did not use opioid analgesia while hospitalized.

Using multivariate regression statistics, it was found that private physicians with residents were 3.1 (95\% $\mathrm{Cl} 1.2-8.4$ ) times more likely to prescribe an opiate prescription at discharge compared with academic physicians with residents $(p=0.02)$. Private physicians without residents were $1.5(95 \% \mathrm{Cl} 0.35$ - 6.6) times more likely to prescribe an opiate at discharge, however, this relationship was not statistically significant. There was no significant difference between the type of provider and the number of tablets prescribed at discharge.

\section{DISCUSSION}

There is mounting evidence that postpartum patients are frequently prescribed excessive amounts of opioid analgesics in the postpartum period. ${ }^{12,13,14}$ The vast majority (97\%) of women with minor perineal trauma after vaginal delivery in this study were prescribed PRN opiates in the inpatient setting. When given an option of as-needed opioid analgesia, the majority of women at $\mathrm{CHH}$ (65.82\%) asked for at least one dose of an opioid analgesic. In comparison, Komatsu et al discovered $31 \%$ of their patients' required opioids for short periods in the hospital, with a median time to opioid cessation of less than one day. ${ }^{15}$ Likewise, a considerably higher percentage of our patients received opioids in comparison to MacArthur et al, who reported opioid use in $7 \%$ of patients who received epidural morphine and $32 \%$ in those who did not receive epidural morphine. ${ }^{16}$ This is contradictory to our finding that women who received epidural analgesia were significantly more likely to use opiates in the inpatient setting $(p=0.009)$.

Even though most of our patients used opioids for analgesia while hospitalized, only a small percentage (8\%) required opioids at the time of discharge. This is similar to the $10 \%$ of patients requiring opioids at discharge as reported by Komatsu et al $^{15}$ as well as Jarlensky et al, who found one in ten Medicaidenrolled women fill an outpatient opioid prescription after vaginal delivery. ${ }^{17}$ Our study differs, however, as our patient sample only represents women experiencing no or minor perineal laceration, while Komatsu et al and Jarlensky et al evaluated opiate prescribing rates for all vaginal deliveries. Prabhu et al found that $29.3 \%$ of patients filled an opiate prescription within seven days of delivery, with only $18 \%$ related to tubal ligation, operative deliveries, or higher-order perineal lacerations. ${ }^{6}$ We describe discharge prescribing practices but did not follow up to see if the prescription was filled. There was no significant difference between opiate use as an inpatient or prescriptions on discharge between types of minor perineal trauma. While Nakahara et al discovered significantly fewer opiates prescribed in the hospital after vaginal delivery to women with first-degree lacerations compared to intact perineum and second-degree lacerations, this may be attributed to small sample size. ${ }^{18}$

Rates of inpatient prescribing and inpatient opiate use were not different among types of practitioners. However, using regression statistical analysis we found patients delivered by private physicians with residents were significantly more likely to receive an opiate prescription at discharge when compared to academic physicians with residents $(\mathrm{OR}=3.1)$. Although the study was conducted at only one delivering facility, midwives, family medicine physicians, non-academic physicians, community physicians working with residents, and full-time academic physicians working with residents represent a diverse group of obstetrical providers included which contributes to the strength of the study. The residents working with both academic physicians and private practice physicians were from the same residency program.

Primiparous women were more likely to use 
inpatient opiates when compared to multiparous women $(p=0.05)$. This is in opposition to Nakahara et al who found that multiparous women were more likely to use narcotics in the postpartum hospitalization period. ${ }^{18}$ Moreover, women who used opiates inpatient were more likely to receive an opiate prescription on discharge $(p=0.005)$. While the majority of women (97\%) were prescribed inpatient as needed use postpartum, there is a risk of women unnecessarily receiving an opiate prescription on discharge. We demonstrated that nearly $3 \%$ of women who did not use opioid analgesia during their hospitalization received a prescription for an opioid at discharge. While opioid dependence is mainly attributable to use in chronic pain management, opioid use for acute pain may be a potential pathway to long-term use. ${ }^{18}$

This study represents a time period during which all medical providers in the state, as a condition of licensure, received education on opioid prescribing best practices. A weakness of our study is the lack of pain description and the amount of inpatient opiate use in response to that pain. Insight into whether the pain was due to perineal and mucosal trauma or due to uterine contractions would have also been beneficial. Lack of breastfeeding status of the mother and how it influenced the choice of analgesic is a study limitation.

\section{CONCLUSION}

Women with no or minor obstetrical lacerations at the time of delivery do experience pain and many will use opioid analgesia if PRN dosing is prescribed while hospitalized. Primiparous women and women who received epidural analgesia in labor were more likely to use opioid analgesia while hospitalized. There was no significant correlation between inpatient opiate use and maternal age, race, gravidity, operative vs. spontaneous delivery, provider status, or type of minor perineal trauma. Women who used opioids as an inpatient were more likely to have opioids prescribed upon discharge. Non-academic physicians with resident coverage were more likely to prescribe opioids at discharge compared to physicians in academic practice. Women receiving opioid analgesic prescriptions at discharge had a higher average maternal age.
Our study set out to describe the characteristics of opioid use for postpartum analgesia in women with no or minor vaginal trauma at the time of delivery. We are hopeful that our findings will fuel future studies looking at larger populations describing the intensity and location of postpartum pain to guide recommendations to achieve appropriate postpartum analgesia.

\section{AUTHOR AFFILIATIONS}

1. Marshall University Joan C. Edwards School of Medicine, Huntington, West Virginia

\section{REFERENCES}

1. Han B, Compton WM, Blanco C, Crane E, Lee J, Jones CM. Prescription opioid use, misuse, and use disorders in U.S. adults: 2015 National Survey on Drug Use and Health. Ann Intern Med. 2017;167:293-301.

2. Centers for Disease Control and Prevention (CDC). Vital signs: overdoses of prescription opioid pain relievers and other drugs among women--United States, 1999-2010. MMWR Morb Mortal Wkly Rep. 2013;62(26):537-542.

3. West Virginia Depart of Health and Human Resources Bureau for Public Health. 2016 West Virginia overdose fatality analysis: healthcare systems utilization, risk factors, and opportunities for Intervention. 2017:10.

4. Guy GP Jr, Zhang K, Bohm MK, et al. Vital signs: changes in opioid prescribing in the United States, 2006-2015. MMWR Morb Mortal Wkly Rep. 2017;66(26):697-704.

5. Paulozzi LJ, Mack KA, Hockenberry JM. Division of Unintentional Injury Prevention, National Center for Injury Prevention and Control, CDC. Vital signs: variation among States in prescribing of opioid pain relievers and benzodiazepines United States, 2012. MMWR Morb Mortal Wkly Rep. 2014;63(26):563-568.

6. Prabhu M, Garry EM, Hernandez-Diaz S, MacDonald SC, Huybrechts KF, Bateman BT. Frequency of opioid dispensing after vaginal delivery. Obstet Gynecol. 2018;132(2):459-465.

7. Lam J, et al. Central nervous system depression of neonates breastfed by mothers receiving 
oxycodone for postpartum analgesia. J Pediatr. 2012;160:33-7.

8. https://www.jointcommission.org/topics/pain management.aspx (downloaded 5/1/2018)

9. Obstetric analgesia and anesthesia. ACOG

Practice Bulletin No. 209. American College of Obstetricians and Gynecologists. Obstet Gynecol 2019;133:e208-25.

10. Chou R, et al. Management of postoperative pain: a clinical practice guideline from the American Pain Society, the American Society of Regional Anesthesia and Pain Medicine, and the American Society of Anesthesiologists' Committee on Regional Anesthesia, Executive Committee, and Administrative Council. J Pain. 2016;17(2):131-157.

11. Wuytack F, et al. Oral non-steroidal antiinflammatory drugs (single dose) for perineal pain in the early postpartum period. Cochrane Database Syst Rev. 2016;7(7):CD011352.

12. Badreldin N, Grobman WA, Chang KT, et al. Opioid prescribing patterns among postpartum women. Am J Obstet Gynecol 2018;219:103.e1-8.

13. Bateman BT, et al. Patterns of opioid prescription and use after cesarean delivery. Obstet Gynecol. 2017;130(1):29-35

14. Osmundson SS, et al. Postdischarge opioid use after cesarean delivery. Obstet Gynecol, 2017;130(1):36-41

15. Komatsu $R$, et al. Recovery after nulliparous birth: a detailed analysis of pain analgesia and recovery of function. Anesthesiology 2017;127(4):684-694.

16. Macarthur $A$, Imarengiaye $C$, Tureanu $L$, Downey K. A randomized, double blind, placebo-controlled trial of epidural morphine analgesia after vaginal delivery. Anesth Analg. 2010;110:159-64

17. Jarlenski, $M$, et al. Filled prescriptions for opioids after vaginal delivery. Obstet Gynecol. 2017; 129(3):431-437.

18. Nakahara A, Ulrich ND, Gala R. Opiate prescribing practices in the postpartum unit. Ochsner Journal. 2018;18:36-41. 